\title{
Caracterización de hongos micorrízicos arbusculares de suelos ganaderos del trópico alto y trópico bajo en Antioquia, Colombia
}

\author{
Characterization of arbuscular mycorrhizal fungi of livestock soils in tropical \\ lowlands and tropical highlands in the department of Antioquia, Colombia
}

\author{
Karla Jaqueline Restrepo Giraldo ${ }^{1}$, María Isabel Montoya Correa ${ }^{1}$, Paula Henao Jaramillo ${ }^{1}$, Lina \\ Andrea Gutiérrez ${ }^{1}$, Licet Paola Molina Guzmán ${ }^{1 *}$
}

\begin{abstract}
RESUMEN
Los hongos micorrízicos arbusculares (HMA) se destacan por su función ecológica de desbloquear y solubilizar los nutrientes del suelo y por su influencia en la estabilidad de los ecosistemas, donde las condiciones edáficas son extremas. En este trabajo se caracterizaron los hongos micorrízicos arbusculares presentes en suelos de sistemas ganaderos del trópico alto y trópico bajo en Antioquia y se exploró la relación entre la cantidad de esporas, el porcentaje de colonización, la diversidad de los géneros de HMA y algunas condiciones físico-químicas del suelo. Se observaron correlaciones entre la densidad de esporas con el Ca $(-0,659), \mathrm{Mg}$ $(-0,715), \mathrm{K}(-0,808), \mathrm{P}(-0,704)$ y pH $(-0,735)$ en Magdalena Medio, así como entre el porcentaje de colonización por HMA y K $(0,719)$ en el Norte de Antioquia. Se determinó la presencia del género Glomus como el más predominante (75-100\%) en las dos subregiones estudiadas, y se encontraron también los géneros Acaulospora spp y Gigaspora spp. en menor cantidad en suelos del trópico alto. Los resultados indican que las características climáticas de las zonas estudiadas y el tipo de pastos que allí prevalecen facilitan el desarrollo y establecimiento de diferentes tipos de micorrizas arbusculares en los distintos suelos y una mayor capacidad para establecer la relación simbiótica con un mayor número de especies de plantas.
\end{abstract}

Palabras clave: esporas, ganadería, micorriza, pastoreo

\begin{abstract}
Arbuscular mycorrhizal fungi (AMF) stand out for their ecological function of unblocking and solubilizing soil nutrients and for their influence on the stability of ecosystems, where edaphic conditions are extreme. In this work, the arbuscular mycorrhizal fungi present in soils of the high tropic and low tropic livestock systems in Antioquia were characterized and the relationship between the number of spores, the colonization percentage, the diversity of the AMF genera and some physical and chemical soil conditions were explored. Correlations were observed between the density of spores with Ca (-0.659), Mg (-0.715), K(-0.808), P (-0.704) and pH (-0.735) in Magdalena Medio, as well as between the percentage of colonization by HMA and $K(0.719)$ in the North of Antioquia. The presence of the genus Glomus was determined as the most predominant (75-100\%) in the two subregions studied. The genera Acaulospora spp and Gigaspora spp were also found in lesser amounts in high topical soils. The results indicate that the climatic characteristics of the zones studied and the type of pastures that prevail there facilitate the development and establishment of different types of arbuscular mycorrhizas to the different soils and a greater capacity to establish the symbiotic relationship with a greater number of species of plants.
\end{abstract}

Key words: spores, livestock, mycorrhizae, grazing

\section{Introducción}

La ganadería es la actividad económica, asociada con la producción pecuaria, más extendida en el mundo, con un tercio de la superficie terrestre destinada al cultivo de pasturas y a la alimentación del ganado (FAO 2017). Esta actividad representa un $88 \%$ del total de la superficie agropecuaria colombiana y en el producto interno bruto tiene una participación cercana al 5\% del total nacional, lo que le da gran importancia a nivel socioeconómico en el país (DANE 2017).

1 Universidad Pontificia Bolivariana, sede Medellín, Facultad de Medicina, Grupo Biología de Sistemas-Calle 78B No. 72A109, Medellín, Colombia.

* Autor para correspondencia: lipa382@gmail.com

Fecha de recepción: 18 julio, 2018.

Fecha de aceptación: 31 octubre, 2018.

DOI: http://dx.doi.org/10.4067/S0718-34292019005000301. Publicado en línea: 11-junio-2019. 
En Colombia se presentan dos tipos de producción de leche: especializada y doble propósito (Calderón et al. 2006). Estas se establecen en diferentes regiones. La lechería especializada se localiza en zonas de trópico alto como el altiplano norte y nordeste de Antioquia, regiones que cuentan con climas frescos a fríos y que están cerca de los centros urbanos. Este sistema se caracteriza por la adaptación de diferentes razas, el uso intensivo de la tierra, el capital y la mano de obra, uso de fertilizantes, riego, rotación de praderas y utilización de suplementos alimenticios (Holmann et al. 2003). El sistema doble propósito se ubica en las zonas de trópico bajo como la Costa Atlántica, valles de los ríos Magdalena, Cauca, Piedemonte Llanero y Caqueteño, en regiones con altas temperaturas y más lejos de los mercados. Se caracteriza por ser una ganadería de tipo extensivo debido a la alta disponibilidad de tierras en estas zonas del país (Calderón et al. 2006). La ganadería bovina colombiana posee un gran reconocimiento, no sólo por sus aportes directos en la alimentación y por todas aquellas actividades derivadas de la transformación de sus productos, sino también por ser uno de los sectores que generan grandes impactos ambientales, como lo son la tala excesiva de árboles, quema de bosques, pérdida de la biodiversidad, desecación de humedales (Lozano et al., 2015). El uso de los suelos para la producción ganadera puede tener efectos, tanto positivos como negativos, sobre la diversidad taxonómica de los microorganismos del suelo; el pisoteo promueve la compactación del suelo, la pérdida de la cobertura vegetal y su erosión, lo que lleva a la disminución de la capacidad de retención de agua y a la infertilidad que puede reducir o agotar los nutrientes necesarios para la óptima producción agrícola. Esto conduce a una pérdida irremediable del suelo. Por otro lado, se sabe que la evacuación de la materia fecal y de la orina de los animales potencia la diversificación de los microorganismos del suelo (Noda 2009).

Entre los microorganismos que se encuentran en el suelo están los hongos formadores de micorrizas conocidos también como hongos micorrízicos arbusculares (HMA), los cuales se destacan por su función ecológica y pueden tener una alta incidencia en la estabilidad de ecosistemas, donde las condiciones edáficas son extremas. La función más importante de las micorrizas es desbloquear y solubilizar los nutrientes del suelo relativamente inmóviles, tales como el zinc (Zn) y el fósforo (P), e igualmente poner los elementos minerales a disposición de la planta para obtener un aumento en su crecimiento. Además le brinda protección contra patógenos, y a cambio de ello la planta le cede carbohidratos y proteínas para su sostenimiento metabólico. La planta y el hongo actúan como simbiontes: juntos son capaces de aumentar hasta un 25\% la producción y no alteran el entorno ambiental. En los ecosistemas débiles y amenazados las micorrizas son las principales cooperadoras para el establecimiento y desarrollo de las plantas (Noda 2009).

En general, se ha señalado que, en las comunidades terrestres, la diversidad de especies vegetales está determinada por la presencia HMA en el suelo; así mismo, se tiene evidencia de que las especies vegetales y las interacciones que estas presentan con los microorganismos del suelo se modifican en presencia de estos hongos. Se ha especificado que las comunidades de HMA muestran diferencias en densidad relativa, producción de esporas y funciones, y a su vez estas diferencias están correlacionadas con el ambiente (temporada de lluvia y sequía, especificidad del hospedero, la presencia de otras especies de hongos, competencia intra e interespecífica y condiciones edafoclimáticas) (Camargo y Esperón 2005).

En Colombia se han desarrollado diferentes estudios que evalúan el efecto de los HMA en cultivos como el café (Bolaños et al. 2000) y se ha analizado la distribución e impacto de estos organismos en suelos amazónicos dedicados a cultivos extensivos; sin embargo, pocos estudios han caracterizado la población de micorrizas asociadas a las condiciones presentes en sistemas de producción ganadera (VallejoQuintero 2013). Este trabajo tuvo como objetivo caracterizar los hongos micorrízicos arbusculares presentes en suelos de sistemas ganaderos del trópico alto y trópico bajo en el departamento de Antioquia, así como explorar la relación entre la cantidad de esporas, el porcentaje de colonización, la diversidad de los géneros de HMA y algunas condiciones físicoquímicas del suelo.

\section{Materiales y métodos}

\section{Descripción de la zona y el tipo de estudio.}

Se realizó un estudio transversal en 48 fincas ganaderas de las subregiones del Norte (trópico alto) y Magdalena Medio (trópico bajo) de Antioquia, en los municipios de Belmira, Entrerríos y San Pedro de los Milagros (Norte de Antioquia), con una temperatura 
que varía entre $13-19^{\circ} \mathrm{C}$, humedad relativa promedio del $79 \%$ y una altura entre $1.850-2.540$ msnm; y en los municipios de Puerto Berrío, Puerto Nare y Puerto Triunfo (Magdalena Medio de Antioquia) con una temperatura entre $32-43^{\circ} \mathrm{C}$, humedad relativa promedio de $53 \%$ y una altura entre $125-150 \mathrm{msnm}$ (IDEAM 2010).

Se diseñó una encuesta para la recolección de información de las fincas. Se registraron datos referentes a la información general del sistema como la distribución y uso de la tierra, tipo de sistema de producción, carga animal, composición de las especies animales de la finca y aspectos relacionados con el uso de fertilizantes en el suelo.

\section{Recolección de muestras de suelo.}

Cada muestra de suelo se recolectó de potreros que se encontraban activos o en pastoreo activo al momento de la visita. Una vez que se definieron los límites de cada potrero, se procedió a tomar submuestras de suelo hasta conformar una "muestra compuesta". Es decir, una muestra de suelo se componía de varias submuestras tomadas aleatoriamente en el potrero (10 submuestras). Para ello se realizó un recorrido sobre el terreno en zig-zag abarcando aproximadamente 2.000 $\mathrm{m}^{2}, \mathrm{y}$ tomando submuestras en cada vértice. En cada punto de submuestreo, con un área de $40 \mathrm{~cm} \mathrm{x} 40 \mathrm{~cm}$, se removieron plantas y hojarasca fresca $(1-3 \mathrm{~cm})$ y se realizó la extracción de suelo a una profundidad de $20 \mathrm{~cm}$. Se transfirieron aproximadamente $200 \mathrm{~g}$ a un recipiente para remover piedras, raíces gruesas, lombrices y artrópodos. La muestra fue desmenuzada y mezclada según lo recomendado por el Instituto Colombiano Agropecuario-ICA (ICA 1992). Por último, se transfirió $1 \mathrm{~kg}$ a una bolsa plástica limpia, cerrada y marcada con el código de la finca muestreada, fecha y municipio.

\section{Análisis físico-químico de suelos.}

Se llevó a cabo usando el equipo de pruebas profesionales para el análisis de suelos modelo STH-14 Código 5010-01 (LaMotte Company, MD, USA). Se evaluó el pH: fuertemente ácido ( $<5.5)$, moderadamente ácido (5.5-6.0), ligeramente ácido (6.1-7.0) y alcalino (<7.0); la concentración de fósforo se estimó por medio de pruebas colorimétricas, mientras que el calcio y potasio se determinaron a través de pruebas de turbidez. Los valores de todos los parámetros se expresaron en mg kg-1, según las recomendaciones del fabricante. Adicionalmente se determinó el contenido de humedad siguiendo el protocolo descrito en la norma ASTM D4959-07 (ASTM International 2007) con algunas modificaciones. La cantidad de suelo fue de $10 \mathrm{~g}$ por muestra y el tiempo de secado de 24 horas.

\section{Caracterización de hongos micorrízicos arbusculares presentes en los suelos}

\section{-Determinación del porcentaje de colonización de hongos micorrízicos arbusculares en raíz.}

Para la determinación del porcentaje de colonización de HMA dentro de la raíz, se realizó una decoloración previa de las raíces con una base fuerte $(\mathrm{KOH} \mathrm{10 \% ).}$ Posteriormente se neutralizó con un ácido $(\mathrm{HCl} 10 \%)$ y se sometieron al colorante azul de tripano al $0.05 \%$ durante 3 días. Luego las raíces fueron observadas en el microscopio (NIKONEclipseE200,USA). Se determinó la presencia de arbúsculos, vesículas y endosporas. Se calculó el porcentaje de colonización en raíz según lo descrito previamente en la literatura (Phillips and Hayman 1970) y el grado de colonización total se estimó usando las siguientes categorías: colonización baja (0$20 \%$ ), colonización media (20,1-50\%) y colonización alta (> 50\%) (Nicholson and Schenck 1979).

\section{Conteo de esporas de hongos micorrízicos arbusculares.}

Para esta determinación se tomaron $10 \mathrm{~g}$ de suelo y se añadieron $20 \mathrm{ml}$ de peróxido de hidrógeno al $5 \%$. Se agitó cada tres minutos durante 15 minutos. Posteriormente se realizó la tamización de las muestras usando tamices de 0.250 y $0.045 \mathrm{~mm}$. Luego se hizo la extracción de las esporas usando un gradiente de centrifugación con sacarosa al $80 \%$, durante 3 minutos a $2.640 \mathrm{rpm}$. Las tres cuartas partes del contenido de los tubos se depositaron en el tamiz de $0.045 \mathrm{~mm}$, se lavó durante 3 minutos para retirar la sacarosa y se transfirió a un papel filtro depositado en una caja de Petri, con el fin de realizar luego el conteo de las esporas en un estereoscopio (NIKON SMZ445, USA). Este resultado fue expresado en número de esporas/g de suelo seco (Pérez and Peroza 2013).

\section{Separación de morfotipos de esporas de hongos micorrízicos arbusculares.}

Después del conteo respectivo de las esporas extraídas de cada muestra, se recolectaron en un vidrio reloj, se observaron al estereoscopio y con 
ayuda de una aguja de disección se reunieron los morfotipos teniendo en cuenta la similitud en forma, color y tamaño de las esporas. Con la ayuda de una micropipeta se extrajeron las esporas correspondientes a cada morfotipo identificado, se rotularon con un código de muestra, asociado a información como la fecha y sitio de recolección, así como la descripción de las características de cada morfotipo encontrado. Finalmente fueron almacenadas a $4^{\circ} \mathrm{C}$ hasta el análisis morfológico de las esporas.

\section{-Identificación de los hongos micorrízicos arbusculares basada en el análisis morfológico de las esporas.}

De cada uno de los morfotipos de esporas se realizó un montaje en placa portaobjetos usando como medio de montaje alcohol polivinílico y ácido láctico glicerol (PVLG) y reactivo de Melzer (Posada et al. 2007). Las esporas fueron evaluadas para su clasificación a nivel de género mediante el uso de claves taxonómicas (Schenck y Pérez 1990) y la clave gráfica publicada por el INVAM- International Culture Collection of Arbuscular Mycorrhizal Fungi (INVAM 2017). Se evaluaron características morfológicas como color, tamaño y forma, asî como las características de la pared (grosor, color y presencia de ornamentaciones).

\section{Plan de análisis.}

Para las variables cuantitativas se calcularon medidas de resumen. Para determinar las relaciones estadísticas entre variables se verificó la normalidad del conjunto de datos con base en la prueba de ShapiroWilk $(p \leq 0,05)$. Posteriormente se estimó el coeficiente de correlación de Spearman (rho), para medir el grado de relación entre el número de esporas y el porcentaje de colonización de HMA respecto a algunas propiedades físico-químicas detectadas por subregión, trópico alto y trópico bajo. Todos los análisis estadísticos se llevaron a cabo en el programa Statistical Package for the Social Sciences (SPSS) versión 22 para Windows XP.

\section{Aspectos éticos.}

Este estudio contó con el aval del Comité de Ética de Investigación en Salud de la Universidad Pontificia Bolivariana (acta $\mathrm{N}^{\circ} 7$ del 23 de mayo de 2012). Se incluyeron las fincas donde los dueños aceptaron participar de manera voluntaria en esta investigación y firmar la autorización para su ejecución .

\section{Resultados y Discusión}

\section{Descripción de las fincas ganaderas estudiadas.}

Las regiones estudiadas presentan condiciones agroclimatológicas contrastantes, altitud y temperatura, generando por ende características ambientales y de manejo técnico particulares en los sistemas de producción de ambas zonas. En las fincas de la subregión Norte (trópico alto) se observó predominancia de la lechería y de la raza Holstein en los sistemas productivos. El tipo de pasto predominante en todos los suelos analizados en las 24 fincas fue el pasto kikuyo (Pennisetum clandestinum). El uso de fertilizantes fue una práctica de manejo existente en todos los predios evaluados, siendo la fertilización química, combinada con la orgánica, la predominante en esta zona (52.2\%), con una periodicidad de fertilización igual o menor a 45 días. En relación con el área, en número de hectáreas (ha), las fincas analizadas no superaron las 300 ha promedio, y de su área total, un 70 a 90\% era utilizada para la ganadería. En cuanto a la carga animal, osciló entre 2 y 4 animales/ha.

En relación con el manejo de los suelos de las fincas de la subregión del Magdalena Medio (trópico bajo), el pasto predominante en todos fue del género Brachiaria, principalmente $B$. decumbes, $B$. humidicula y B. brizantha. Los sistemas de producción principales fueron la cría y el doble propósito. El uso de fertilizantes no fue una práctica de manejo del suelo reportada por los productores de esta zona. En cuanto al área de las fincas, estas se destacaron por ser explotaciones de gran tamaño. En general, superaban las 800 ha promedio, y de su área total, un 80 a $100 \%$ era utilizada para la ganadería, donde la carga animal oscilaba entre 3 y 6 bovinos/ ha. Con respecto a las razas presentes en los sistemas de producción evaluados en esta zona, se observó predominio de la raza Cebú y el mestizaje con ganado Normando, Pardo suizo y algunos que debido a su amplio proceso de cruzamientos o heterosis han sido denominados criollos colombianos.

\section{Características físico-químicas de los suelos.}

En relación con las propiedades físico-químicas de los suelos de las fincas en estudio, en ambas regiones se observó que la concentración de humus, nitrito, potasio, aluminio y nitrógeno amoniacal fue constante en todos los municipios evaluados (bajo, $1 \mathrm{mg} \mathrm{kg}^{-1}$, $50 \mathrm{mg} \mathrm{kg}^{-1}, 86 \mathrm{mg} \mathrm{kg}^{-1} \mathrm{y} 5 \mathrm{mg} \mathrm{kg}^{-1}$, respectivamente). 
Con respecto a los valores de $\mathrm{pH}$ obtenidos en los suelos de los municipios de la subregión Norte, se observó que estos varían entre moderadamente y ligeramente ácidos (5.5-7.0). Caso contrario ocurrió en los municipios de la subregión de Magdalena Medio, donde los valores de $\mathrm{pH}$ de las fincas evaluadas fueron catalogados como fuertemente ácidos, es decir, valores de $\mathrm{pH}<5.5$.

Con relación a la concentración de calcio, se observó que en la subregión Norte fue mayor, con un promedio de $3.458 \mathrm{mg} \mathrm{kg}^{-1}$, con respecto a la detectada en la subregión de Magdalena Medio, donde se halló una concentración promedio de $2.791 \mathrm{mg} \mathrm{kg}^{-1}$. Caso contrario ocurrió al evaluar la concentración promedio de fósforo y magnesio, donde en los suelos de las fincas de la subregión del Magdalena Medio se observaron valores mayores ( $67 \mathrm{mg} \mathrm{kg}^{-1}$ y $30 \mathrm{mg} \mathrm{kg}^{-1}$, respectivamente), en comparación con la concentración observada en los suelos de los municipios de la subregión Norte (29 $\mathrm{mg} \mathrm{kg}^{-1}$ y $12 \mathrm{mg} \mathrm{kg}^{-1}$, respectivamente).

En cuanto al porcentaje de humedad, se observó que es mayor en los suelos de los municipios ubicados en la zona Norte, en relación con los suelos de la subregión del Magdalena Medio. En promedio la humedad en los suelos ubicados en las fincas ganaderas de San Pedro de los Milagros fue de 85\%, Entrerríos $73 \%$, Belmira 68\%, mientras que en Puerto Triunfo fue de 25\%, Puerto Nare 20\% y Puerto Berrio $18 \%$.

Se ha señalado previamente que los suelos ubicados en las zonas tropicales poseen una alta dependencia micorrízica. Sin embargo, la colonización de estos está determinada por diversas condiciones, tales como factores relacionados con la especie de planta (pasto) y su régimen de explotación, la eficiencia de los géneros de HMA para colonizar la planta hospedera y las condiciones físico-químicas del suelo, ya sea pH, temperatura, aireación, textura y contenido de materia orgánica, condiciones climáticas, humedad, épocas de lluvia y sequía y las prácticas agronómicas, culturales y de aplicación de pesticidas. Estas condiciones pueden incidir en la efectividad y el funcionamiento de esta simbiosis, y de hecho, en el caso de los sistemas ganaderos, podrían influir en la productividad del pasto (González et al. 2007).

Se ha evidenciado que los HMA, cuando se unen con distintas especies de gramíneas, favorecen la absorción de agua y la transferencia de nutrientes, como el fósforo, magnesio, nitrógeno, potasio, calcio, azufre, zinc. Además, se ha determinado que estimulan el crecimiento de las plantas y mejoran la producción, la calidad nutricional e incrementan la tolerancia de las especies de pasto al pisoteo, especialmente en suelos de baja y moderada fertilidad, ayudando finalmente a la recuperación rápida de estos, después de la defoliación hecha por los animales herbívoros (Guerra y Chacón 2012).

Se ha establecido que bajas concentraciones de calcio pueden afectar la síntesis de proteínas en las plantas, ocasionando un menor desarrollo radical y poco crecimiento de los tallos y hojas. En cuanto a la concentración de magnesio, valores bajos de este elemento han sido observados comúnmente en suelos bajo condiciones de intensa lluvia, así como en suelos con $\mathrm{pH}$ moderadamente bajo, donde la solubilidad del magnesio disminuye y se hace menos disponible (Pérez et al. 2016). Los datos obtenidos en las fincas de ambas zonas concuerdan con las observaciones reportadas previamente en la literatura, donde la concentración de calcio es menor en suelos del trópico bajo y la concentración de magnesio es mayor en los suelos del trópico bajo, situación que se evidencia también en los suelos con vocación ganadera, como los analizados en el presente estudio.

\section{Porcentaje de colonización de HMA en raíz.}

Al evaluar el porcentaje promedio de colonización de HMA en raíz, en los suelos de las fincas en estudio, se encontró que todas presentaron un valor en el rango de la categoría de porcentaje medio de colonización de HMA (20-50\%). No obstante, al analizar la variabilidad individual de este dato entre las ocho fincas de cada municipio y en cada subregión, se observó amplia variabilidad en el trópico alto, con valores que oscilaron entre 11 y $91 \%$ y en el trópico bajo entre 4.0 y $86 \%$.

En las fincas de la subregión Norte, en el municipio de Entrerríos y Belmira, se observó colonización de HMA entre el rango de categoría baja y alta (0$20 \%$ y $>50$, respectivamente), con porcentajes de colonización bajo de 11 y 17\%, respectivamente, con una diferencia significativa $(\mathrm{p}=0.05)$ con respecto a las demás fincas. En el municipio de San Pedro de los Milagros se encontró que el porcentaje de colonización detectado en las fincas estuvo en la categoría baja y media (0-20\% y 20.1-50\%, respectivamente), donde el mayor porcentaje fue de $51 \%$ y el menor de $13 \%$. Los resultados para las fincas de este municipio son estadísticamente similares entre ellas $(\mathrm{p}>0.05)$ (Figura 1).

Con relación a la subregión del Magdalena Medio, se observó que la colonización de HMA en las fincas 

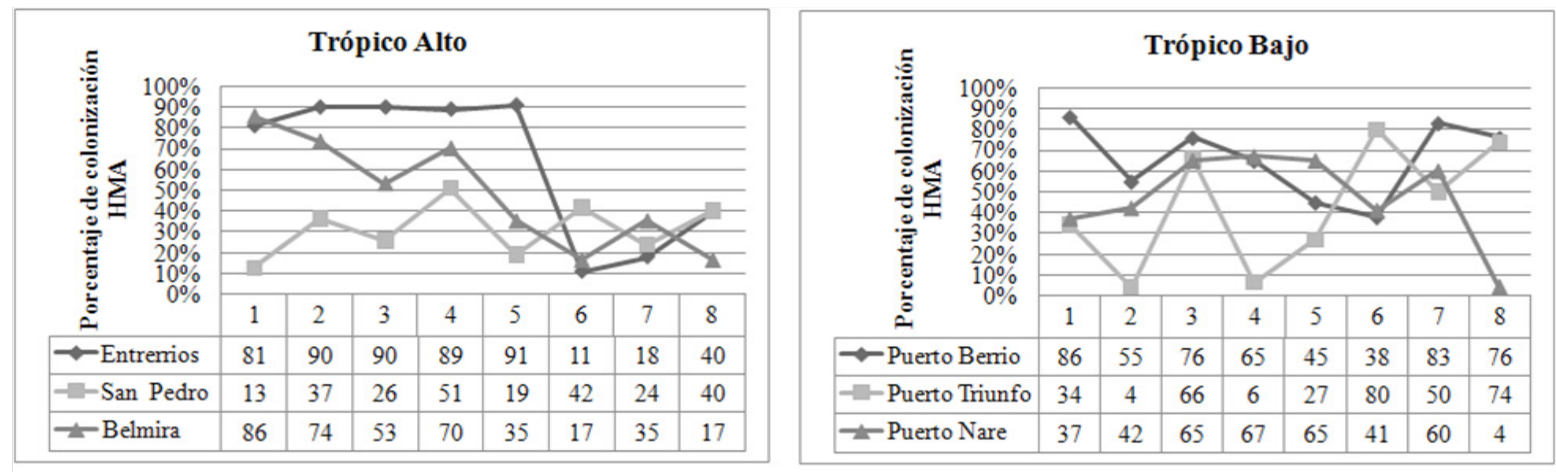

Figura 1. Variabilidad del porcentaje (\%) de colonización de Hongos Micorrízicos Arbusculares (HMA) por fincas ganaderas de cada municipio ubicado en trópico alto (A) y trópico bajo (B).

del municipio de Puerto Berrío estuvo en la categoría media y alta (20.1-50\% y $>50$, respectivamente), con porcentaje de 86 y $83 \%$, ( $p=0.03)$. En el municipio de Puerto Triunfo el porcentaje de colonización estuvo entre la categoría baja y alta $(0-20 \%$ y $>50$, respectivamente), donde el mayor porcentaje fue de $80 \%$ (columna 6 figura 1- B) en relación con lo observado en la finca F34 (columna 2Figura 1-B) con un $4 \%$ ( $\mathrm{p}=0.01$ ). Resultados similares fueron encontrados en el municipio de Puerto Nare, donde el porcentaje de colonización de HMA en la finca F44 (columna 4 figura 1-B) fue de 67\% y en la finca F48 (columna 8 Figura 1-B) de 4\%, con una diferencia significativa $(\mathrm{p}=0.001)$ (Figura 1$)$.

Autores como Pérez et al. (2012) han sugerido que el porcentaje mínimo de colonización de HMA debe ser superior al $40 \%$ para que favorezca la relación simbiótica con la planta y sirva como indicador biológico de la calidad del suelo. Por lo tanto, los porcentajes de colonización de las fincas muestreadas se encontraban principalmente en la categoría media de 20-50\%, pudiendo constituir un buen referente para su posterior utilización como inoculo fúngico. Pérez y Peroza (2013) reportaron valores entre un 20-76\%, con un promedio de $42 \%$ de colonización de HMA en el municipio de Tolú, Sucre, en fincas ganaderas de zonas agrológicas diferentes según el uso del suelo, con predominio de pasto Angleton y caracterizadas por presentar $\mathrm{pH}$ de muy ácido a fuertemente ácido. Posada et al (2007) encontraron en dos zonas del municipio de Florencia Caquetá con predominancia de pasto Brachiaria decumbens, un porcentaje de colonización de HMA del $74.8 \%$, resultados similares a los obtenidos en este estudio para ese tipo de pasto. En relación con los valores de porcentaje de colonización de las raíces con los HMA que se detectaron en sistemas de trópico alto en los cuales predominaba el pasto kikuyo (Penisetum clandestinum), estos fueron mayores en comparación con los pastos de trópico bajo. Estos resultados podrían deberse al uso de fertilizantes que favorecieron una mayor asociación de los hongos con la planta en la zona de trópico alto, dato contrastante con lo reportado por Noda (2009), quien sugirió que la fertilización y el tipo de inoculo micorrízico tienen influencia positiva sobre el porcentaje de asociación.

\section{Recuento de esporas de HMA}

Al evaluar la densidad poblacional de esporas de HMA detectada en los suelos de cada subregión, se observó que el mayor número se halló en los suelos de las fincas ubicadas en los municipios de la subregión Norte de Antioquia. En el municipio de Entrerríos se encontró un valor promedio de 363 esporas/g de suelo seco, seguido por los suelos del municipio de Belmira y San Pedro de los Milagros con 216 y 203 esporas/g de suelo seco, respectivamente. En los suelos de las fincas ganaderas ubicadas en los municipios de la subregión del Magdalena Medio se observó que el municipio de Puerto Nare presentó el mayor recuento de esporas, con 80 esporas/g de suelo seco, seguido por el municipio de Puerto Triunfo (56 esporas/g de suelo seco) y el municipio de Puerto Berrio con 51 esporas/g de suelo seco (Figura 2).

\section{Identificación morfológica de las esporas de HMA encontradas en los suelos de trópico alto y bajo en Antioquia.}

De cada muestra se tomó un total de 10 esporas para su identificación por medio de las claves taxonómicas, donde se encontraron tres géneros predominantes de HMA en la rizosfera de las 48 fincas en estudio (Figura 3). La distribución y abundancia de géneros se observa en la Tabla 1. 


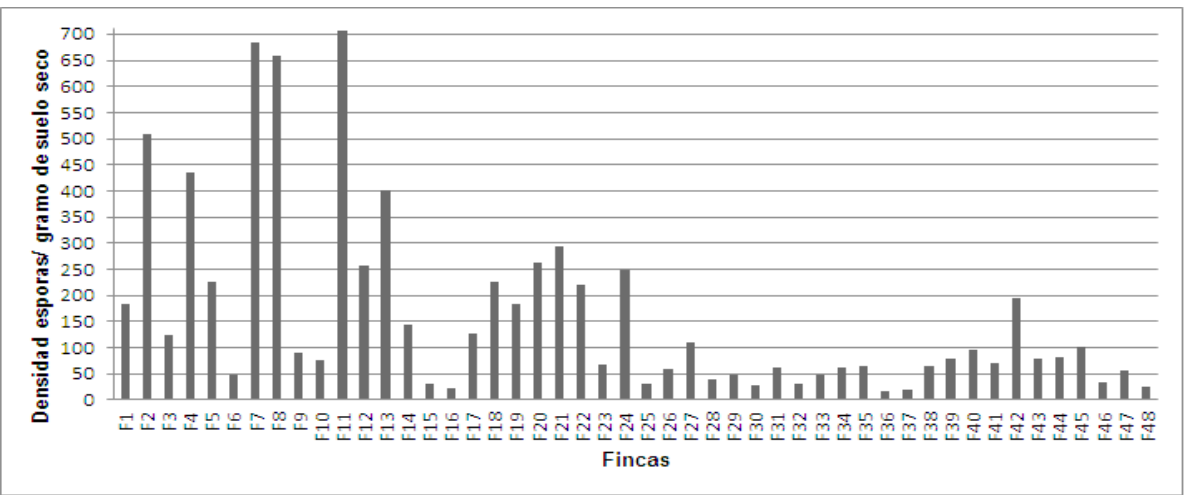

Figura 2. Densidad de número de esporas por gramo de suelo seco en dos subregiones de Antioquia. F1-F8: Fincas del municipio de Entrerríos, F9-F16: Fincas del municipio de Belmira, F17-F24: Fincas municipio de San Pedro de Los Milagros, F25-F32: Fincas del municipio de Puerto Berrio, F33-F40: Fincas del municipio de Puerto Triunfo, F40-F48: Fincas municipio de Puerto Nare.
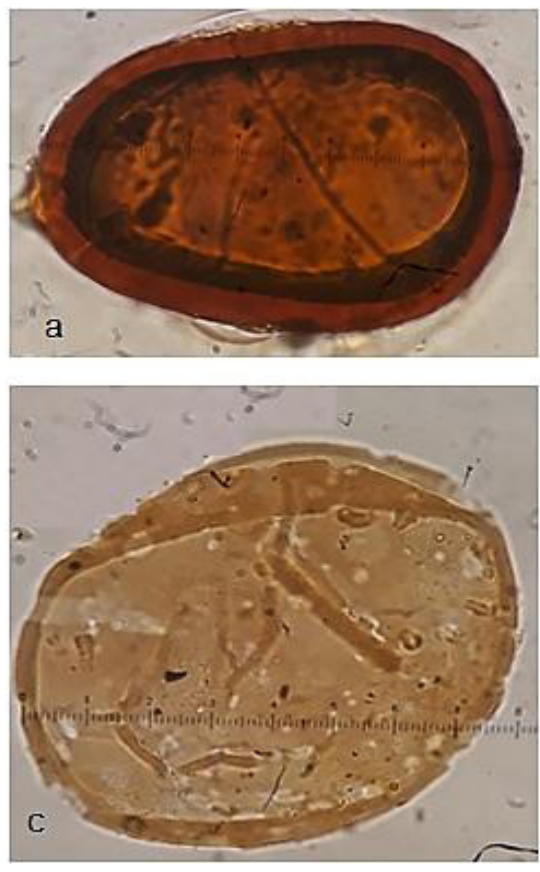

Figura 3. Géneros de HMA identificados a partir del análisis morfológico de las esporas obtenidas en las diferentes muestras de suelos evaluadas en la subregión Norte y Magdalena de Antioquia. a: Glomus spp, b: Gigaspora spp, c y d: Acaulospora spp.

Tabla 1. Distribución porcentual (\%) de los géneros de HMA identificados en los suelos de los sistemas ganaderos evaluados en trópico alto y trópico bajo. $n=80$

\begin{tabular}{cccc}
\hline Municipios trópico Alto & Glomus $(\%)$ & Acualospora $(\%)$ & Gigaspora $(\%)$ \\
\hline Entrerrios & 75 & 12,5 & 12,5 \\
Belmira & 75 & 25 & - \\
San Pedro de los Milagros & 100 & - & - \\
\hline Municipios trópico Bajo & 100 & - & - \\
Puerto Berrío & 100 & - & - \\
Puerto Triunfo & 95 & 5 & - \\
Puerto Nare &
\end{tabular}


El HMA detectado en los suelos estudiados, con mayor abundancia y amplia distribución geográfica, fue del género Glomus. Esto concuerda con los resultados reportados previamente por otros autores, quienes han registrado mayor predominancia de este género de HMA en diversidad de suelos (Guerra $y$ Chacón. 2012). En este sentido, se ha señalado que el género Glomus posee capacidad para adaptarse a diferentes condiciones edáficas y una mayor capacidad para establecer la relación simbiótica con un amplio número de especies de plantas en Colombia. En un estudio previo desarrollado en el departamento del Guaviare se halló un porcentaje de 55\%, con relación a la abundancia de esporas nativas del género Glomus, en comparación con los géneros Acaulospora (27\%), Entrophospora (9\%) y Scutellospora (9\%), asociados con pasturas de Brachiaria decumbens (Gonzalez etal., 2007). Otros autores han señalado que en el pasto Brachiaria sp., en el piedemonte del Departamento de Caquetá, se han observado porcentajes de colonización de $60 \%$ con el género Glomus, seguido por el hongo Acaulospora spp. con un 20\% (Posada et al 2007).

Los resultados obtenidos en el presente estudio sugieren que el género de HMA Glomus posee mejor capacidad de adaptación a las condiciones edáficas de los suelos dedicados a la actividad ganadera, tanto en el trópico alto como bajo, y se encuentra en mayor abundancia y ampliamente distribuido en estas zonas ecológicamente contrastantes. Sin embargo, se sabe que el efecto del hospedero sobre el establecimiento de las poblaciones de HMA puede variar de acuerdo con la dependencia micorrízica de la planta, en combinación con las condiciones edáficas del suelo. En este caso, los datos de diversidad y abundancia de los géneros de HMA observados en los suelos de ambas zonas, podrían deberse a una dependencia contrastante entre los dos tipos de pastos predominantes en cada una de las zonas estudiadas. En este trabajo solo se hizo la identificación taxonómica de los HMA hasta el nivel de género. Sería recomendable la realización de estudios moleculares posteriores para confirmar estos hallazgos y llegar hasta la caracterización de especies, que permitan establecer con mayor precisión el comportamiento de estos hongos en los diferentes sistemas productivos y su relación con los niveles de calidad del suelo.

\section{Análisis de la correlación entre el recuento de esporas, el porcentaje de colonización y las propiedades físico-químicas de los suelos estudiados.}

Para los valores de concentración de elementos como el Ca, Mg, K y P y pH obtenidos en este estudio, se encontró una correlación negativa significativa fuerte entre estos parámetros y el recuento de esporas para la subregión de Magdalena Medio. No obstante, en la subregión del Norte, la correlación fue negativa significativa leve para la concentración de calcio. Para los demás parámetros evaluados no se encontró correlación. Adicionalmente, al analizar la correlación de los parámetros con el porcentaje de colonización, se observó una correlación positiva leve con la concentración de fósforo y potasio en la subregión del Norte, mientras que para los demás parámetros evaluados y la subregión de Magdalena Medio, no se halló correlación alguna.

Una concentración de Ca y $\mathrm{Mg}$ alta resulta tóxica para la biota del suelo, por lo tanto, es de esperar un número reducido de esporas (Cabrales et al. 2016). $\mathrm{Al}$ evaluar la concentración promedio de fósforo y magnesio en los suelos de las fincas de la subregión del Magdalena Medio, se detectaron valores mayores (67 $\mathrm{mg} \mathrm{kg}^{-1}$ y $30 \mathrm{mg} \mathrm{kg}^{-1}$, respectivamente), con respecto a la concentración en los suelos de los municipios de la subregión Norte $\left(29 \mathrm{mg} \mathrm{kg}^{-1}\right.$ y 12 , respectivamente). La correlación negativa con el $\mathrm{K}$ coincide con lo reportado por Pérez y Peroza (2013), quienes observaron este tipo de correlación en suelos dedicados a la ganadería en el municipio de Tolú (Sucre). La influencia del K en el número de esporas se debe probablemente a que las concentraciones de este elemento en el suelo fueron altas $\left(50 \mathrm{mg} \mathrm{kg}^{-1}\right)$ para el $97,5 \%$ de las fincas muestreadas, lo que explica que al tener el suelo mayores niveles de este elemento, menor es la cantidad de esporas encontradas en la rizosfera. En el caso del P, cuanto mayor fue el contenido en el suelo, menor fue la cantidad de esporas. Estos datos confirman lo obtenido por Pérez et al., 2016, donde se determinó que al incrementar la concentración del P en el suelo, la conductividad eléctrica aumenta y modifica los potenciales osmóticos que pueden incidir, tanto en la diversidad como en el número de esporas (Tabla 2). 
Tabla 2. Análisis de correlación de Spearman (Rho) entre el número de esporas y el porcentaje de colonización de HMA con algunas propiedades físico-químicos del suelo en las fincas de ganadería de trópico alto y trópico bajo estudiadas

\begin{tabular}{|c|c|c|c|c|c|c|c|c|c|c|}
\hline & \multicolumn{2}{|c|}{$\mathrm{pH}$} & \multicolumn{2}{|c|}{ Fósforo (mg kg-1) } & \multicolumn{2}{|c|}{ Potasio (mg kg-1) } & \multicolumn{2}{|c|}{ Calcio (mg kg-1) } & \multicolumn{2}{|c|}{$\begin{array}{l}\text { Magnesio } \\
\left(\mathrm{mg} \mathrm{kg}^{-1}\right)\end{array}$} \\
\hline & Norte & $\begin{array}{l}\text { Magdalena } \\
\text { medio }\end{array}$ & Norte & $\begin{array}{l}\text { Magdalena } \\
\text { medio }\end{array}$ & Norte & $\begin{array}{l}\text { Magdalena } \\
\text { medio }\end{array}$ & Norte & $\begin{array}{c}\text { Magdalena } \\
\text { medio }\end{array}$ & Norte & $\begin{array}{c}\text { Magdalena } \\
\text { medio }\end{array}$ \\
\hline $\begin{array}{l}\text { Esporas/g } \\
\text { suelo }\end{array}$ & $0,530 * *$ & $-0,735^{*}$ & $-0,272$ & $-0,703^{*}$ & $0,312 *$ & $-0,807 * *$ & $-0,48 * *$ & $-0,659 *$ & $0,49 * *$ & $-0,715^{*}$ \\
\hline $\begin{array}{l}\text { Porcentaje } \\
\text { colonización } \\
\text { de HMA } \\
\text { en raíz }\end{array}$ & $-0,208$ & 0,370 & $-0,550 * *$ & $-0,770$ & $0,428 * *$ & 0,650 & $-0,266$ & 0,214 & $-0,133$ & 0,230 \\
\hline
\end{tabular}

* La correlación es significativa al nivel de p<0,05, **La correlación es significativa al nivel de p<0,001

En cuanto al porcentaje de colonización y la concentración de $\mathrm{K}$, se observó una correlación positiva leve (Tabla 2). Esto a su vez sugiere que es diferente la relación que tienen las esporas presentes en el suelo con las concentraciones de bases, a la absorción de nutrientes a través del micelio, ya que para el segundo caso se hace referencia a la dinámica del K en el suelo. Se concluye que la planta mejora su sistema de transporte de este elemento a través de la colonización de las raíces por parte del micelio intramatricial de los HMA (Pérez et al. 2016).

En relación con el valor de $\mathrm{pH}$ obtenido en los suelos de las fincas ubicadas en los municipios de la subregión Norte, se observó que son moderadamente ácidos, contrario a lo que se encontró en la subregión del Magdalena Medio, donde los valores de $\mathrm{pH}$ fueron fuertemente ácidos. El efecto del $\mathrm{pH}$ es directo sobre la solubilidad y disponibilidad de elementos para las raíces de las plantas, incluyendo fósforo, hierro, manganeso, cobre, zinc y aluminio. Además la respuesta de los HMA al pH del suelo puede ser variable, aunque se considera que los HMA pueden tolerar condiciones adversas de $\mathrm{pH}$ por modificación de la micorrizósfera durante el proceso de toma de nutrientes (Pérez et al. 2011).

Asímismo, al considerar la subregión de Magdalena Medio como un terreno con montañas de poca elevación con procesos de erosión laminar, sometidos al uso en ganadería extensiva, se evidencian muy bajos niveles de biomasa microbiana e índice de respiración. Adicionalmente, es posible que haya conteos muy altos de esporas micorrícicas, ya que el $\mathrm{pH}$ fuertemente ácido tiende a favorecer el desarrollo de los hongos, en este caso formadores de micorrizas arbusculares y la producción de sus estructuras reproductivas (González et al. 2007).

\section{Conclusiones}

En el caso de los sistemas ganaderos en el departamento de Antioquia, aún no se cuenta con suficiente información sobre los géneros de esporas de HMA presentes en los suelos y la influencia de estos según su distribución y el tipo de pastos que allí se observan. Esta investigación proporciona una descripción inicial sobre la diversidad de géneros encontrados en fincas ganaderas del Norte (trópico alto) y Magdalena Medio (trópico bajo).

En conjunto, todos los datos obtenidos sugieren que las características climáticas contrastantes de las zonas estudiadas y el tipo de pastos que allí prevalecen están facilitando el desarrollo y establecimiento de diferentes tipos de HMA, lo cual tiene un efecto benéfico sobre el suelo. Además evidencia que el género Glomus está presentando mayor adaptación a los diferentes suelos y mayor capacidad para establecer la relación simbiótica con un mayor número de especies de plantas.

\section{Agradecimientos}

ACOLETRIUNFO, APAGRONAR-Asociación de Productores Agropecuarios de Puerto Nare, COREGAN -Comité Regional de Ganaderos de Puerto Berrio y COLANTA, propietarios, personal administrativo y técnico de las fincas estudiadas por su invaluable cooperación.

Además, los autores agradecen el financiamiento al Departamento Administrativo de Ciencia, Tecnología e Innovación COLCIENCIAS, en el marco del proyecto con código 121056934576, contrato 6532013, Radicado CIDI UPB 211B-02/14-65. 


\section{Literatura citada}

\section{ASTM International}

2007. ASTM D4959-07, standard test method for determination of water (moisture) content of soil by direct heating. Disponible en: https://www.astm.org/. Consultado: 18/feb/2016.

Bolaños, B.M.M.; Rivillas O.C.A.; Suárez V.S.

2000. Identificación de micorrizas arbusculares en suelos de la zona cafetera colombiana. Cenicafé, 51 (4): 245-262.

Cabrales, E.; Toro, M.; López, D.

2016. Efecto de micorrizas nativas yfósforo en los rendimientos del maíz en Guárico. Temas Agrarios, 21 (2): 21-31.

Calderón A., García, F.; Martínez, G.

2006. Indicadores de calidad de leches crudas en diferentes regiones de Colombia. MVZ Córdoba, 11 (1): 725-37.

Camargo, S.; Esperón, M.

2005. Efecto de la heterogeneidad espacial y estacional del suelo sobre la abundancia de esporas de hongos micorrizógenos arbusculares en el valle semiárido de Tehuacán-Cuicatlán, México. Revista de Biología Tropical, 53 (3-4): 339-52.

\section{DANE.}

2017. Producto Interno Bruto (PIB)-2017/2. Disponible en: http://www.dane.gov.co/index.php/52-espanol/noticias/ noticias/4282-producto-interno-bruto-pib-ii-trimestre-2017. Consulted: 27/sep/2017.

González, P.J.; Fernández, D.; Plana, R.; Crespo, G.

2007. Efectos del antecedente cultural en las micorrizas nativas y la productividad del pasto brachiaria (Brachiaria decumbens cv. Señal). Pastos y Forrajes, 30 (1): 1.

Guerra, S.B.E.; Chacón, M.R.

2012. Simbiosis micorrízica arbuscular y acumulación de aluminio en Brachiaria decumbensy Manihot esculenta. Rev.Bio.Agro, 10 (2): 87-98.

Holmann, F.; Rivas, L.; Carulla, J.; Giraldo, L.; Guzmán, S.; Martínez, M.; Rivera, B.; Medina, A.; Farrow, A.

2003. Evolución de los sistemas de producción de leche en el trópico latinoamericano y su interrelación con los mercados: un análisis del caso colombiano. Centro Internacional de Agricultura Tropical (CIAT), International Livestock Research Institute (ILRI) and Systemwide Livestock Program (SLP). Documento de Trabajo \# 193. Cali (Colombia): CIAT. Disponible en: http://hdl.handle.net/10568/1288 Consultado: 30/jul/2018.

IDEAM.

2010. Catálogo de Estaciones Hidrometeorológicas. Disponible: http://www.ideam.gov.co Consultado: 30/jul/2018.

Instituto Colombiano Agropecuario (ICA).

1992. Fertilización en diversos cultivos: Quinta aproximación. Santa fé de Bogotá: Centro de Investigación Tibaitatá. 64 p. INVAM.

2017. "International Culture Collection of Arbuscular and Vesicular Mycorrhizal Fungi.” Disponible en: http://fungi. invam.wvu.edu/. Consultado: 22/sep/2017.
Lozano, S.J.; Armbrecht, I.; Montoya, L.J.

2015. Hongos formadores de micorrizas arbusculares y su efecto sobre la estructura de los suelos en fincas con manejos agroecológicos e intensivos. Acta Agronómica, 64 (4): 289-296.

Nicolson, T., Schenck, N.

1979. Endogonaceous Mycorrhizal Endophytes in Florida. Mycologia, 71 (1): 178-198.

Noda, Yolai.

2009. Las Micorrizas: Una alternativa de fertilización ecológica en los pastos. Pastos y Forrajes, 32 (2): 1.

Organización de las Naciones Unidas para la Alimentación y la Agricultura-FAO.

2017. Producción animal. Disponible en: http://www.fao.org/ animal-production/es/. Consulted: 30/jun/2018.

Pérez, A., Cury, K., Oviedo, L.

2016. Colonización de micorrizas arbusculares en tres especies de pasturas del departamento de Sucre. Temas Agrarios, 21 (2): 65-75.

Pérez, A.; Peroza, V.

2013. Micorrizas Arbusculares asociadas al pasto Angleton (Dichathium Aristatum Benth) en fincas ganaderas del municipio de Tolú, Sucre-Colombia. Rev. MVZ Córdoba, 18 (1): 3362-69.

Pérez, A.; Rojas, J.; Montes, D.

2011. Hongos formadores de micorrizas arbusculares: una alternativa biológica para la sostenibilidad de los agroecosistemas de praderas en el Caribe colombiano. Revista Colombiana Ciencia Animal, 3 (2): 366-85.

Pérez-Luna,Y., Álvarez-Solís, J.D., Mendoza-Vega, J., PatFernández, J.M., Gómez-Álvarez, R., Cuevas, L.

2012. Diversidad de hongos micorrícicos arbusculares en maíz con cultivo de cobertura y biofertilizantes en Chiapas, México.Gayana. Botánica, 69 (1): 46-56.

Phillips, J.M.; Hayman D.S.

1970. Improved procedures for clearing roots and staining parasitic and vesicular-arbuscular mycorrhizal fungi for rapid assessment of infection. Transactions of the British Mycological Society, 55 (1): 158-IN18.

Posada, R.; Franco, L.; Cuéllar, A.; Sánchez, W. 2007. Inoculum of Arbuscular Mycorrhizal Fungi on Brachiaria decumbens (Poaceae) Pastures in Valley and Hilly Terrain. Acta de Biología Colombiana, 12 (1): 113-19.

Schenck, N.; Pérez, Y.

1990. Manual for the identification of VA mycorrhizal fungi. Synergistic Publications. Gainesville, FL: 286 p.

Vallejo-Quintero, V. E.

2013. Importancia y utilidad de la evaluación de la calidad de suelos mediante el componente microbiano: experiencias en sistemas silvopastoriles. Colombia Forestal, 16 (1): 83-99. 